\title{
ON FOURIER COEFFICIENTS OF SIEGEL MODULAR FORMS OF DEGREE TWO WITH RESPECT TO CONGRUENCE SUBGROUPS
}

\author{
MASATAKA CHIDA, HIDENORI KATSURADA, KOHJI MATSUMOTO
}

\begin{abstract}
We prove a formula of Petersson's type for Fourier coefficients of Siegel cusp forms of degree 2 with respect to congruence subgroups, and as a corollary, show upper bound estimates of individual Fourier coefficient. The method in this paper is essentially a generalization of Kitaoka's previous work which studied the full modular case, but some modification is necessary to obtain estimates which are sharp with respect to the level aspect.
\end{abstract}

\section{INTRODUCTION AND THE STATEMENT OF MAIN RESULTS}

Let $M_{l}(R)$ be the set of $l \times l$ matrices whose components belong to a ring $R$, and define

$$
\begin{aligned}
& \Lambda=\left\{S \in \mathrm{M}_{2}(\mathbb{Z}) \mid{ }^{t} S=S\right\}, \\
& \Lambda^{*}=\left\{S=\left(s_{i j}\right) \in \mathrm{M}_{2}(\mathbb{Q}) \mid s_{i i} \in \mathbb{Z}, 2 s_{12}=2 s_{21} \in \mathbb{Z}\right\} .
\end{aligned}
$$

Let $\mathbb{H}_{2}=\left\{Z=X+\left.i Y \in M_{2}(\mathbb{C})\right|^{t} Z=Z, Y>0\right\}$ be the Siegel upper half space of degree 2, $\operatorname{Sp}(2, \mathbb{Z})$ be the Siegel modular group of degree 2 which consists of all non-singular matrices $M \in M_{4}(\mathbb{Z})$ satisfying ${ }^{t} M J M=J$, where $J=\left(\begin{array}{cc}1_{2} \\ -1_{2}\end{array}\right)$. For a positive integer $N$, define

$$
\Gamma_{0}^{(2)}(N)=\left\{\left(\begin{array}{ll}
A & B \\
C & D
\end{array}\right) \in \operatorname{Sp}(2, \mathbb{Z}) \mid C \equiv\left(\begin{array}{ll}
0 & 0 \\
0 & 0
\end{array}\right) \bmod N\right\} .
$$

Denote by $S_{k}\left(\Gamma_{0}^{(2)}(N)\right)$ the space of Siegel cusp forms of weight $k(\geq 1)$ with respect to $\Gamma_{0}^{(2)}(N)$, and write the Fourier expansion of $f \in S_{k}\left(\Gamma_{0}^{(2)}(N)\right)$ as

$$
f(Z)=\sum_{0 \leq Q \in \Lambda^{*}} a_{f}(Q) e(\operatorname{tr}(Q Z)),
$$

where $Z \in \mathbb{H}_{2}, e(x)=\exp (2 \pi i x)$ and tr denotes the trace map. The main purpose of the present paper is to prove a Petersson type formula for the above Fourier coefficients $a_{f}(Q)$. Such a study was carried out by Kitaoka [5] in the full modular case, in order to obtain an upper bound estimate of Fourier coefficients. Our motivation is to generalize Kitaoka's result to the case of general $\Gamma_{0}(N)$.

First recall the classical elliptic modular case. Let $S_{k}\left(\Gamma_{0}(N)\right)$ be the space of elliptic cusp forms of weight $k$ and level $N, f \in S_{k}\left(\Gamma_{0}(N)\right)$, and denote its Fourier coefficients by $a_{f}(n)$. Then the classical Petersson formula is

$$
\begin{aligned}
& \frac{\Gamma(k-1)}{(4 \pi \sqrt{m n})^{k-1}} \sum_{f} \frac{\overline{a_{f}(m)} a_{f}(n)}{\langle f, f\rangle} \\
& =\delta_{m n}+\frac{2 \pi}{(-1)^{k / 2}} \sum_{\substack{c>0 \\
c \equiv 0 \text { (modN })}} \frac{1}{c} S(m, n ; c) J_{k-1}\left(\frac{4 \pi \sqrt{m n}}{c}\right),
\end{aligned}
$$

Key words and phrases. Siegel modular form, Fourier coefficients, Petersson formula. 
where $\langle$,$\rangle denotes the usual Petersson inner product, \delta_{m n}$ is the Kronecker delta, $S(m, n ; c)$ is the Kloosterman sum, $J_{k-1}(\cdot)$ is the $(k-1)$-th $J$-Bessel function, and the sum on the left-hand side runs over an orthogonal basis of $S_{k}(N)$ (see Theorem 3.6 in [3]). From this formula, evaluating the sum on the right-hand side, we can show

$$
\frac{\Gamma(k-1)}{(4 \pi \sqrt{m n})^{k-1}} \sum_{f} \frac{\overline{a_{f}(m)} a_{f}(n)}{\langle f, f\rangle}=\delta_{m n}+O\left((m, n)^{1 / 2}(m n)^{(k-1) / 2} N^{1 / 2-k} d(N)\right),
$$

where $d(\cdot)$ denotes the divisor function (Duke [1], Kamiya [4]).

Now return to the Siegel case. Let $\mathcal{F}_{k, N}$ be a set of orthogonal basis of $S_{k}\left(\Gamma_{0}^{(2)}(N)\right)$. For $Q, T \in \Lambda^{*}$, define

$$
\delta(Q, T)=\#\left\{U \in \mathrm{GL}(2, \mathbb{Z}) \mid U Q^{t} U=T\right\} .
$$

In what follows, $Q$ is to be regarded as fixed, and $\varepsilon$ denotes an arbitrarily small positive number, not necessarily the same at each occurrence. The constants implied by Landau's $O$-symbol and Vinogradov's « symbol may depend on $Q, \varepsilon$.

Now we state our main results in the present paper.

Theorem 1.1. Let $Q, T \in \Lambda^{*}$, both are positive definite. Then

(i) We have

$$
\begin{gathered}
\pi^{1 / 2}(4 \pi)^{3-2 k} \Gamma\left(k-\frac{3}{2}\right) \Gamma(k-2)(\operatorname{det} Q)^{-k+\frac{3}{2}} \sum_{f \in \mathcal{F}_{k, N}} \frac{\overline{a_{f}(Q)} a_{f}(T)}{\langle f, f\rangle} \\
=\delta(Q, T)+E_{Q}(T, N),
\end{gathered}
$$

where $E_{Q}(T, N)$ is the error term, in the sense that it tends to 0 when $N \rightarrow \infty$. Moreover the estimates

(ii) $E_{Q}(T, N)=O\left(N^{\frac{3}{2}-k}|T|^{k-\frac{3}{2}}+N^{2-k+\varepsilon}|T|^{\frac{k}{2}-\frac{1}{4}+\varepsilon}+N^{3-2 k+\varepsilon}|T|^{k-1+\varepsilon}\right)$,

(iii) $E_{Q}(T, N)=O\left(N^{-1 / 2+\varepsilon}|T|^{k / 2-1 / 4+\varepsilon}\right)$

hold for $k \geq 3$.

Remark 1.2. (i) When there is no $U \in \mathrm{GL}(2, \mathbb{Z})$ satisfying $U Q^{t} U=T$, obviously $\delta(Q, T)=0$. Therefore the role of $\delta(Q, T)$ is similar to the delta symbol in formula (1.3).

(ii) This result is a generalization of Proposition 3.3 in Kowalski-Saha-Tsimerman [7]. They applied the estimate to show an equidistribution result for $L$-functions associated to Siegel cusp forms of genus 2 and growing weight $k$. So it is expected that our result can be used to prove a similar result for growing level $N$.

From the above theorem, as we will see in the next section, we can deduce an upper bound estimate of individual Fourier coefficient.

Theorem 1.3. When there is no $U \in \mathrm{GL}(2, \mathbb{Z})$ satisfying $U Q^{t} U=T$, we obtain

(a) $a_{f}(T)=O\left(N^{\frac{3}{2}-k}|T|^{k-\frac{3}{2}}+N^{2-k+\varepsilon}|T|^{\frac{k}{2}-\frac{1}{4}+\varepsilon}+N^{3-2 k+\varepsilon}|T|^{k-1+\varepsilon}\right)$.

(b) $a_{f}(T)=O\left(N^{-1 / 2+\varepsilon}|T|^{k / 2-1 / 4+\varepsilon}\right)$

for $k \geq 3$.

When $N=1$, Theorem 1.3 (b) is exactly Kitaoka's estimate [5]. However, the estimate with respect to $N$ is rather weak in (b). This point is supplied by (a), which gives a sharp estimate with respect to $N$. This (a) corresponds to the error estimate of Duke-Kamiya in (1.3).

In the following sections we will give the proof of the above theorems. Many parts of the proof are rather straightforward generalizations of Kitaoka's argument in [5], but we describe the details because we have to trace carefully how is the effect of $N$. In particular, some 
modification of Kitaoka's argument is necessary to obtain estimates which are sharp with respect to $N$.

\section{PoINCARÉ SERIES}

For $M=\left(\begin{array}{ll}A & B \\ C & D\end{array}\right) \in \operatorname{Sp}(2, \mathbb{Z})$ and $Z \in \mathbb{H}_{2}$, we set

$$
j(M, Z)=\operatorname{det}(C Z+D)
$$

and

$$
M\langle Z\rangle=(A Z+B)(C Z+D)^{-1}
$$

Moreover, we set

$$
\Gamma_{1}^{(2)}(\infty)=\left\{\left(\begin{array}{cc}
1_{2} & S \\
& 1_{2}
\end{array}\right) \mid S \in \Lambda\right\} .
$$

For $Q \in \Lambda^{*}$ with $Q>0$ and positive integers $k, N$, we define the Poincaré series $g_{N}(Z, Q)$ of weight $k$ with respect to $\Gamma_{0}^{(2)}(N)$ by

$$
g_{N}(Z, Q)=\sum_{M \in \Gamma_{1}^{(2)}(\infty) \backslash \Gamma_{0}^{(2)}(N)} e(\operatorname{tr}(Q \cdot M\langle Z\rangle)) j(M, Z)^{-k} .
$$

For $f, g \in S_{k}\left(\Gamma_{0}^{(2)}(N)\right)$, we define the (unnormalized) Petersson norm of $f$ and $g$ by

$$
\langle f, g\rangle=\int_{\Gamma_{0}^{(2)}(N) \backslash \mathbb{H}_{2}} f(Z) \overline{g(Z)}(\operatorname{det} Y)^{k-3} d Z .
$$

Proposition 2.1. Let $f \in S_{k}\left(\Gamma_{0}^{(2)}(N)\right)$. Then we have

$$
\left\langle g_{N}(\cdot, Q), f\right\rangle=\pi^{1 / 2}(4 \pi)^{3-2 k} \Gamma\left(k-\frac{3}{2}\right) \Gamma(k-2)(\operatorname{det} Q)^{-k+\frac{3}{2}} \overline{a_{f}(Q)},
$$

and consequently,

$$
g_{N}(Z, Q)=\pi^{1 / 2}(4 \pi)^{3-2 k} \Gamma\left(k-\frac{3}{2}\right) \Gamma(k-2)(\operatorname{det} Q)^{-k+\frac{3}{2}} \sum_{f \in \mathcal{F}_{k, N}} \frac{\overline{a_{f}(Q)} f(Z)}{\langle f, f\rangle} .
$$

Proof. This is a direct generalization of a result in Klingen's book [6], page 90. We briefly outline the argument. We follow the argument in pp.76-90 of Klingen [6] with replacing $\Gamma_{n}$ and $A_{n}$ by $\Gamma_{0}^{(2)}(N)$ and $\Gamma_{1}^{(2)}(\infty)$, respectively. The starting point, Proposition 1 ([6, p.76]), does not depend on $N$. Formulas (7), (8) in [6, p.78] are proved by the technique of decomposing the Siegel half space $H_{n}$ into copies (by the action of $\{ \pm 1\} \backslash \Gamma_{n}$ ) of the fundamental domain $F_{n}$. The same technique can be applied to our present situation, with replacing $S_{n}^{k}$ by $S_{n, k}(N)$. In this way, we follow Klingen's argument until Proposition 3 ([ 6 , p.85]). In the statement of Proposition 3 , the series $G_{n}^{k}\left(z ; g_{\nu}\right)$ is defined, but this is again independent of $N$. (But be careful with the definition of $\Lambda_{n}$.) Also $N$ does not appear in the Fourier expansion of $f \in S_{n, k}(N)$. On the last line of p.87, Klingen defines $A_{n}$, which differs from our $\Gamma_{1}^{(2)}(\infty)$ by the factor 2 . Therefore, $g_{n}^{k}(z, t)$ defined on p.90 of [6] differs from our $g_{N}(Z, T)$ by the factor 2 . All other parts of the proof are the same as in [6]. 
Substituting (1.1) (with replacing $Q$ by $T$ ) into the right-hand side of (2.2), we have

$$
\begin{aligned}
g_{N}(Z, Q) & =\pi^{1 / 2}(4 \pi)^{3-2 k} \Gamma\left(k-\frac{3}{2}\right) \Gamma(k-2)(\operatorname{det} Q)^{-k+\frac{3}{2}} \\
& \times \sum_{0 \leq T \in \Lambda^{*}} \sum_{f \in \mathcal{F}_{k, N}} \frac{\overline{a_{f}(Q)} a_{f}(T)}{\langle f, f\rangle} e(\operatorname{tr}(T Z)) .
\end{aligned}
$$

Therefore, if we write the Fourier expansion of the Poincaré series as

$$
g_{N}(Z, Q)=\sum_{0 \leq T \in \Lambda^{*}} A_{Q, N}(T) e(\operatorname{tr}(T Z))
$$

we obtain

$$
A_{Q, N}(T)=\pi^{1 / 2}(4 \pi)^{3-2 k} \Gamma\left(k-\frac{3}{2}\right) \Gamma(k-2)(\operatorname{det} Q)^{-k+\frac{3}{2}} \sum_{f \in \mathcal{F}_{k, N}} \frac{\overline{a_{f}(Q)} a_{f}(T)}{\langle f, f\rangle} .
$$

Therefore the Fourier coefficient $A_{Q, N}(T)$ can be estimated by our Theorem 1.1. In particular, when there is no $U \in \mathrm{GL}(2, \mathbb{Z})$ satisfying $U Q^{t} U=T$, from Theorem 1.1 (ii), (iii) (with noting Remark 1.2) we find that $A_{Q, N}(T)$ satisfies the estimations stated in Theorem 1.3. Since any cusp form can be written as a linear combination of Poincaré series, we obtain the assertion of Theorem 1.3 .

On the other hand, (2.5) implies that, in order to prove Theorem 1.1, it is enough to consider $A_{Q, N}(T)$.

Let $\mathfrak{H}_{N}$ be a complete system of representatives of $\Gamma_{1}^{(2)}(\infty) \backslash \Gamma_{0}^{(2)}(N) / \Gamma_{1}^{(2)}(\infty)$. For $M \in$ $\operatorname{Sp}(2, \mathbb{Z})$, we denote

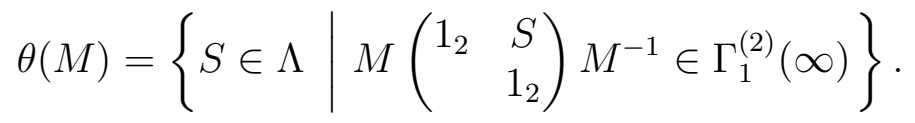

Lemma 2.2 (Kitaoka [5], p.158, Lemma 1). For $M \in \operatorname{Sp}(2, \mathbb{Z})$, we have

$$
\Gamma_{1}^{(2)}(\infty) M \Gamma_{1}^{(2)}(\infty)=\coprod_{S \in \Lambda / \theta(M)} \Gamma_{1}^{(2)}(\infty) M\left(\begin{array}{cc}
1_{2} & S \\
& 1_{2}
\end{array}\right)
$$

From this lemma, it is easy to see that

$$
g_{N}(Z, Q)=\sum_{M \in \mathfrak{H}_{N}} H_{Q}(M, S)
$$

where

$$
H_{Q}(M, Z)=\sum_{S \in \Lambda / \theta(M)} e(\operatorname{tr}(Q \cdot M\langle Z+S\rangle)) j(M, Z+S)^{-k} .
$$

Write the Fourier expansion of $H_{Q}(M, Z)$ as

$$
H_{Q}(M, Z)=\sum_{0 \leq T \in \Lambda^{*}} h_{Q}(M, T) e(\operatorname{tr}(T Z)) .
$$

Then

$$
h_{Q}(M, T)=\int_{X(\bmod 1)} H_{Q}(M, Z) e(-\operatorname{tr}(T Z)) d X
$$

where $X=\left(\begin{array}{ll}x_{1} & x_{2} \\ x_{2} & x_{4}\end{array}\right)=\Re(Z), d X=d x_{1} d x_{2} d x_{4}$. 
Comparing (2.6), (2.8) with (2.4), we obtain

$$
A_{Q, N}(T)=\sum_{M \in \mathfrak{H}_{N}} h_{Q}(M, T) .
$$

Therefore, to prove Theorem 1.1, our remaining task is to evaluate each term on the righthand side of (2.10). Let

$$
\mathfrak{H}_{N}^{(i)}=\left\{M=\left(\begin{array}{cc}
A & B \\
C & D
\end{array}\right) \in \mathfrak{H}_{N} \mid \operatorname{rank} C=i\right\}
$$

for $i=0,1$ or 2 , and decompose (2.10) as

$$
A_{Q, N}(T)=\Sigma_{0}+\Sigma_{1}+\Sigma_{2}
$$

where

$$
\Sigma_{i}=\sum_{M \in \mathfrak{H}_{N}^{(i)}} h_{Q}(M, T) \quad(i=0,1,2) .
$$

In the following three sections we evaluate $\Sigma_{0}, \Sigma_{1}$ and $\Sigma_{2}$, respectively.

3. THE CASE OF $\operatorname{rank} C=0$

In this section, we assume $\operatorname{rank} C=0$, i.e. $C=0$.

Lemma 3.1 (Kitaoka [5], p.158, Lemma 3). As $\mathfrak{H}_{N}^{(0)}$ we can choose

$$
\left\{\left(\begin{array}{cc}
{ }^{t} U & 0 \\
0 & U^{-1}
\end{array}\right) \mid U \in \mathrm{GL}(2, \mathbb{Z})\right\}
$$

and $\theta(M)=\Lambda$.

Proposition 3.2. We have

$$
\Sigma_{0}=\#\left\{U \in \mathrm{GL}(2, \mathbb{Z}) \mid U Q^{t} U=T\right\},
$$

which is hence non-zero only if $Q \sim T$.

Proof. We can choose $M$ which is of the form stated in Lemma 3.1, and $\theta(M)=\Lambda$. Hence from (2.7) we have

$$
H_{Q}(M, Z)=e(\operatorname{tr}(Q \cdot M\langle Z\rangle)) j(M, Z)^{k}=e\left(\operatorname{tr}\left(Q \cdot{ }^{t} U Z U\right)\right) .
$$

Therefore (2.9) gives

$$
h_{Q}(M, T)=\int_{X \bmod 1} e\left(\operatorname{tr}\left(Q \cdot{ }^{t} U Z U\right)\right) e(-\operatorname{tr}(T Z)) d X .
$$

Then we have

$$
\begin{aligned}
\sum_{M \in \mathfrak{H}_{N}^{(0)}} h_{Q}(M, T) & =\sum_{U \in \mathrm{GL}(2, \mathbb{Z})} \int_{X \bmod 1} e\left(\operatorname{tr}\left(Q \cdot{ }^{t} U Z U\right)\right) e(-\operatorname{tr}(T Z)) d X \\
& =\sum_{U \in \mathrm{GL}(2, \mathbb{Z})} \int_{0}^{1} \int_{0}^{1} \int_{0}^{1} e\left(\operatorname{tr}\left(Q \cdot{ }^{t} U Z U-T Z\right)\right) d x_{1} d x_{2} d x_{4},
\end{aligned}
$$

where

$$
X=\left(\begin{array}{ll}
x_{1} & x_{2} \\
x_{2} & x_{4}
\end{array}\right)=\Re(Z) .
$$

Since $\operatorname{tr}\left(Q^{t} U Z U-T Z\right)=0$ is equivalent to $\operatorname{tr}\left(\left(U Q^{t} U-T\right) Z\right)=0$, we see that if $U Q^{t} U=T$ then $\operatorname{tr}\left(Q^{t} U Z U-T Z\right)=0$ for all $Z$, hence the above integral is equal to 1 . On the other 
hand, if $U Q^{t} U \neq T$, then $\operatorname{tr}\left(Q^{t} U Z U-T Z\right) \neq 0$ for almost all $Z$, so the above integral vanishes. This completes the proof.

\section{THE CASE OF $\operatorname{rank} C=1$}

Next we consider the case $\operatorname{rank} C=1$.

Lemma 4.1. As $\mathfrak{H}_{N}^{(1)}$ we can choose

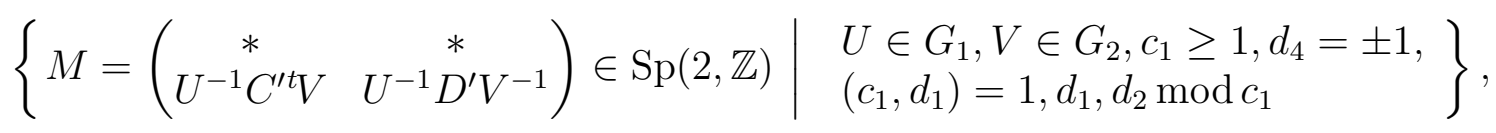

where

$$
C^{\prime}=\left(\begin{array}{cc}
N c_{1} & 0 \\
0 & 0
\end{array}\right), D^{\prime}=\left(\begin{array}{cc}
d_{1} & d_{2} \\
0 & d_{4}
\end{array}\right), G_{1}=\left\{\left(\begin{array}{ll}
* & * \\
0 & *
\end{array}\right)\right\} / \operatorname{GL}(2, \mathbb{Z}), G_{2}=\operatorname{GL}(2, \mathbb{Z})\left\{\left(\begin{array}{ll}
1 & * \\
0 & *
\end{array}\right)\right\} .
$$

Moreover $\theta(M)$ is given by

$$
\left\{\begin{array}{l|l}
S \in \Lambda & \left.S[V]=\left(\begin{array}{ll}
0 & 0 \\
0 & *
\end{array}\right)\right\}
\end{array}\right.
$$

for the above specialized $M$, where $S[V]={ }^{t} V S V$.

When $N=1$, this is Kitaoka's Lemma 4 ([5], p.159). The above generalization is obvious. For $U, V$ in the setting of Lemma 4.1, we set

$$
P=\left(\begin{array}{cc}
p_{1} & p_{2} / 2 \\
p_{2} / 2 & p_{4}
\end{array}\right)=Q\left[{ }^{t} U\right]=U Q^{t} U
$$

and

$$
S=\left(\begin{array}{cc}
s_{1} & s_{2} / 2 \\
s_{2} / 2 & s_{4}
\end{array}\right)=T\left[{ }^{t} V^{-1}\right]=V^{-1} T^{t} V^{-1} .
$$

We choose an $a_{1}$ satisfying $a_{1} d_{1} \equiv 1 \bmod c_{1}$. The following is Kitaoka's Lemma 1 ([5], p.160) when $N=1$.

Lemma 4.2. With the notation as above, we have

$$
\begin{aligned}
h_{Q}(M, T) & =(-1)^{k / 2} \sqrt{2} \pi|Q|^{\frac{3}{4}-\frac{k}{2}}|T|^{\frac{k}{2}-\frac{3}{4}} \delta_{p_{4}, s_{4}} s_{4}^{-\frac{1}{2}}\left(N c_{1}\right)^{-\frac{3}{2}} \\
& \times e\left(\frac{a_{1} s_{4} d_{2}^{2}-\left(a_{1} d_{4} p_{2}-s_{2}\right) d_{2}}{N c_{1}}+\frac{a_{1} p_{1}+d_{1} s_{1}}{N c_{1}}+\frac{d_{4} p_{2} s_{2}}{2 N c_{1} s_{4}}\right) J_{k-\frac{3}{2}}\left(\frac{4 \pi \sqrt{|T||Q|}}{N c_{1} s_{4}}\right) .
\end{aligned}
$$

Using this lemma, we evaluate $\Sigma_{1}$. First recall

$$
\sum_{n \bmod c} e\left(\frac{a n^{2}+b n}{c}\right)=O\left((a, c)^{\frac{1}{2}} c^{\frac{1}{2}}\right) .
$$

This is a well-known estimate on generalized quadratic Gauss sums, but here we give a sketch of proof. Denote the left-hand side by $G(a, b, c)$. When $(a, c)>1$, then $G(a, b, c)=0$ unless $(a, c) \mid b$, while in the latter case

$$
G(a, b, c)=(a, c) G(a /(a, c), b /(a, c), c /(a, c)),
$$

so we may reduce the problem to the case $(a, c)=1$. When $(a, c)=1$, we write $c=u v$, where $u$ is a power of 2 and $v$ is odd. The decomposition $G(a, b, c)=G(a u, b, v) G(a v, b, u)$ holds, and $G(a u, b, v)$ is explicitly written in the form $\eta v^{\frac{1}{2}}$, where $\eta$ is a certain complex number with $|\eta|=1$. Applying Theorem 10.1 of Hua [2, Chapter 7], we find $G(a v, b, u)=O\left(u^{\frac{1}{2}}\right)$ (here, the $\varepsilon$-factor in Hua's statement is not necessary because now $u$ has only one prime divisor). Therefore $G(a, b, c)=O\left(c^{\frac{1}{2}}\right)$ as desired. 
Using (4.1), we find

$$
\sum_{d_{2} \bmod N c_{1}} e\left(\frac{a_{1} s_{4} d_{2}^{2}-\left(a_{1} d_{4} p_{2}-s_{2}\right) d_{2}}{N c_{1}}\right)=O\left(\left(a_{1} s_{4}, N c_{1}\right)^{\frac{1}{2}}\left(N c_{1}\right)^{\frac{1}{2}}\right)=O\left(\left(s_{4}, N c_{1}\right)^{\frac{1}{2}}\left(N c_{1}\right)^{\frac{1}{2}}\right)
$$

where the last equality follows from the fact $\left(a_{1}, N c_{1}\right)=1$. Therefore

$$
\left|\sum_{d_{2} \bmod N c_{1}} h_{Q}(M, T)\right| \ll \delta_{p_{4}, s_{4}}|T|^{\frac{k}{2}-\frac{3}{4}} s_{4}^{-\frac{1}{2}}\left(N c_{1}\right)^{-1}\left(s_{4}, N c_{1}\right)^{\frac{1}{2}}\left|J_{k-\frac{3}{2}}\left(\frac{4 \pi \sqrt{|T||Q|}}{N c_{1} s_{4}}\right)\right|
$$

(because $Q$ is fixed and so the $Q$-factor is to be included in the implied constant), which further implies

$$
\begin{aligned}
& \sum_{U \in G_{1}} \sum_{\substack{d_{1} \bmod N c_{1} \\
\left(d_{1}, N c_{1}\right)=1, d_{4}= \pm 1}}\left|\sum_{d_{2}} h_{Q}(M, T)\right| \\
& \ll \sum_{U \in G_{1}} \delta_{p_{4}, s_{4}}\left|T c^{\frac{k}{2}-\frac{3}{4}} s_{4}^{-\frac{1}{2}}\left(s_{4}, N c_{1}\right)^{\frac{1}{2}}\right| J_{k-\frac{3}{2}}\left(\frac{4 \pi \sqrt{|T||Q|}}{N c_{1} s_{4}}\right) \mid .
\end{aligned}
$$

Since $G_{1}$ is parametrized by the second row up to sign, we see that the right-hand side of (4.2) is

$$
\ll \sum_{u=\left(\begin{array}{l}
u_{3} \\
u_{4}
\end{array}\right)} \delta_{p_{4}, s_{4}}|T|^{\frac{k}{2}-\frac{3}{4}} s_{4}^{-\frac{1}{2}}\left(s_{4}, N c_{1}\right)^{\frac{1}{2}}\left|J_{k-\frac{3}{2}}\left(\frac{4 \pi \sqrt{|T||Q|}}{N c_{1} s_{4}}\right)\right|
$$

where $u_{3}, u_{4}$ is determined by $U=\left(\begin{array}{ll}u_{1} & u_{2} \\ u_{3} & u_{4}\end{array}\right)$. Since $P=Q\left[{ }^{t} U\right]$, we have

$$
Q[u]=\left(u_{3} u_{4}\right) Q\left(\begin{array}{l}
u_{3} \\
u_{4}
\end{array}\right)=p_{4}
$$

Therefore $\delta_{p_{4}, s_{4}}=\delta_{Q[u], s_{4}}$, but $\#\left\{u \mid Q[u]=s_{4}\right\}=O\left(s_{4}^{\varepsilon}\right)$. Hence (4.3) is

$$
\ll|T|^{\frac{k}{2}-\frac{3}{4}} s_{4}^{-\frac{1}{2}+\varepsilon}\left(s_{4}, N c_{1}\right)^{\frac{1}{2}}\left|J_{k-\frac{3}{2}}\left(\frac{4 \pi \sqrt{|T||Q|}}{N c_{1} s_{4}}\right)\right| .
$$

Therefore

$$
\begin{aligned}
\sum_{V \in G_{2}} & \sum_{U \in G_{1}} \sum_{\substack{d_{1} \bmod N c_{1} \\
\left(d_{1}, N c_{1}\right)=1, d_{4}= \pm 1}}\left|\sum_{d_{2} \bmod N c_{1}} h_{Q}(M, T)\right| \\
& \ll \sum_{V \in G_{2}}|T|^{\frac{k}{2}-\frac{3}{4}} s_{4}^{-\frac{1}{2}+\varepsilon}\left(s_{4}, N c_{1}\right)^{\frac{1}{2}}\left|J_{k-\frac{3}{2}}\left(\frac{4 \pi \sqrt{|T||Q|}}{N c_{1} s_{4}}\right)\right| .
\end{aligned}
$$

We see easily that $V$ is parametrized by the first column, and $s_{4}=T\left(\begin{array}{c}-v_{3} \\ v_{1}\end{array}\right)$ for $V=$ $\left(\begin{array}{ll}v_{1} & v_{2} \\ v_{3} & v_{4}\end{array}\right)$. Therefore, setting

$$
A(m, T)=\#\left\{\left(\begin{array}{l}
v_{1} \\
v_{2}
\end{array}\right) \mid\left(v_{1}, v_{2}\right)=1, T\left(\begin{array}{l}
v_{1} \\
v_{2}
\end{array}\right)=m\right\}
$$


we find that the right-hand side of (4.5) is

$$
\ll|T|^{\frac{k}{2}-\frac{3}{4}} \sum_{s_{4}=1}^{\infty} s_{4}^{-\frac{1}{2}+\varepsilon}\left(s_{4}, N c_{1}\right)^{\frac{1}{2}} A\left(s_{4}, T\right)\left|J_{k-\frac{3}{2}}\left(\frac{4 \pi \sqrt{|T||Q|}}{N c_{1} s_{4}}\right)\right| .
$$

Here we quote the following well-known estimates:

$$
J_{k-\frac{3}{2}}(x)= \begin{cases}\text { (i) } & O\left(x^{k-\frac{3}{2}}\right) \\ \text { (ii) } & O\left(x^{-\frac{1}{2}}\right)\end{cases}
$$

for $x>0$ (see Kitaoka [5], p.163, Lemma 2). Applying (4.7) (i), we see that (4.6) is

$$
\begin{aligned}
& \ll|T|^{\frac{k}{2}-\frac{3}{4}} \sum_{s_{4}=1}^{\infty} s_{4}^{-\frac{1}{2}+\varepsilon}\left(s_{4}, N c_{1}\right)^{\frac{1}{2}} A\left(s_{4}, T\right)\left(\frac{4 \pi \sqrt{|T||Q|}}{N c_{1} s_{4}}\right)^{k-\frac{3}{2}} \\
& \ll|T|^{k-\frac{3}{2}} \sum_{s_{4}=1}^{\infty} A\left(s_{4}, T\right) s_{4}^{-\frac{1}{2}+\varepsilon} s_{4}^{\frac{1}{2}}\left(N c_{1} s_{4}\right)^{-k+\frac{3}{2}} .
\end{aligned}
$$

Finally, since $\sum_{c_{1}=1}^{\infty} c_{1}^{-k+\frac{3}{2}}<+\infty$ (if $k>\frac{5}{2}$ ), we have

$$
\begin{aligned}
\Sigma_{1} & =\sum_{c_{1}=1}^{\infty} \sum_{V \in G_{2}} \sum_{U \in G_{1}} \sum_{\substack{d_{1} \bmod N c_{1} \\
\left(d_{1}, N c_{1}\right)=1, d_{4}= \pm 1}} \sum_{d_{2} \bmod N c_{1}} h_{Q}(M, T) \\
& \ll|T|^{k-\frac{3}{2}} \sum_{c_{1}=1}^{\infty} \sum_{s_{4}=1}^{\infty} A\left(s_{4}, T\right) s_{4}^{-k+\frac{3}{2}+\varepsilon} N^{-k+\frac{3}{2}} c_{1}^{-k+\frac{3}{2}} \\
& \ll|T|^{k-\frac{3}{2}} N^{-k+\frac{3}{2}} \sum_{s_{4}=1}^{\infty} A\left(s_{4}, T\right) s_{4}^{-k+\frac{3}{2}+\varepsilon} .
\end{aligned}
$$

Since $A\left(s_{4}, T\right)=O\left(s_{4}^{\varepsilon}\right)$ (independent of $T$ ), we now arrive at the following proposition.

Proposition 4.3. If $k \geq 3$, then

$$
\Sigma_{1} \ll|T|^{k-\frac{3}{2}} N^{\frac{3}{2}-k} .
$$

This proposition is necessary for the proof of assertion (ii) of Theorem 1.1. To prove assertion (iii) of Theorem 1.1, we have to modify the above argument, using the both estimates of (4.7). That is, to evaluate the Bessel factor in (4.6), now we apply (4.7) (ii) if $4 \pi \sqrt{|T||Q|} \geq N c_{1} s_{4}$, and apply (i) if $4 \pi \sqrt{|T||Q|}<N c_{1} s_{4}$. Then (4.6) is

$$
\ll|T|^{\frac{k}{2}-\frac{3}{4}}\left(S_{1}+S_{2}\right),
$$

where

$$
\begin{aligned}
S_{1} & =\sum_{s_{4} \leq \tau / N c_{1}} s_{4}^{-\frac{1}{2}+\varepsilon}\left(s_{4}, N c_{1}\right)^{\frac{1}{2}} A\left(s_{4}, T\right)|T|^{-\frac{1}{4}}\left(N c_{1} s_{4}\right)^{\frac{1}{2}} \\
S_{2} & =\sum_{s_{4}>\tau / N c_{1}} s_{4}^{-\frac{1}{2}+\varepsilon}\left(s_{4}, N c_{1}\right)^{\frac{1}{2}} A\left(s_{4}, T\right)|T|^{\frac{k}{2}-\frac{3}{4}}\left(N c_{1} s_{4}\right)^{-k+\frac{3}{2}}
\end{aligned}
$$

with $\tau=4 \pi \sqrt{|T||Q|}$. Therefore

$$
\Sigma_{1} \ll|T|^{\frac{k}{2}-\frac{3}{4}} \sum_{c_{1}=1}^{\infty}\left(S_{1}+S_{2}\right) .
$$


Lemma 4.4. We have

$$
\begin{gathered}
\sum_{1 \leq N c_{1} \leq \tau / s_{4}}\left(s_{4}, N c_{1}\right)^{\frac{1}{2}}\left(N c_{1}\right)^{\frac{1}{2}} \ll N^{-\frac{1}{2}+\varepsilon}\left(\frac{\tau}{s_{4}}\right)^{\frac{3}{2}} s_{4}^{\varepsilon}, \\
\sum_{N c_{1}>\tau / s_{4}}\left(s_{4}, N c_{1}\right)^{\frac{1}{2}}\left(N c_{1}\right)^{\frac{3}{2}-k} \ll N^{-\frac{1}{2}+\varepsilon}\left(\frac{\tau}{s_{4}}\right)^{\frac{5}{2}-k} s_{4}^{\varepsilon} \quad(k \geq 3) .
\end{gathered}
$$

Proof. When $N=1$, this is Kitaoka's Lemma 2 ([5], p.163). As for (4.9), first we write $\left(s_{4}, N c_{1}\right)=r$ and $N c_{1}=r q$ to obtain

$$
\sum_{1 \leq N c_{1} \leq \tau / s_{4}}\left(s_{4}, N c_{1}\right)^{\frac{1}{2}}\left(N c_{1}\right)^{\frac{1}{2}}=\sum_{r \mid s_{4}} r \sum_{q \leq \tau / s_{4} r, N \mid q r} q^{\frac{1}{2}} .
$$

Put $(N, r)=\nu$, and write $N=\nu N^{\prime}$. Then $N \mid q r$ implies $N^{\prime} \mid q$, so we can write $q=N^{\prime} q^{\prime}$. Therefore the above is

$$
\begin{aligned}
& =\sum_{r \mid s_{4}} r \sum_{q^{\prime} \leq \tau / s_{4} r N^{\prime}}\left(N^{\prime} q^{\prime}\right)^{\frac{1}{2}} \\
& \ll \sum_{r \mid s_{4}} r \frac{1}{N^{\prime}}\left(\frac{\tau}{s_{4} r}\right)^{\frac{3}{2}} \\
& =\left(\frac{\tau}{s_{4}}\right)^{\frac{3}{2}} \sum_{\nu \mid N} \frac{1}{N^{\prime}} \sum_{r \mid s_{4}, r \equiv 0(\bmod \nu)} r^{-\frac{1}{2}} \\
& \leq\left(\frac{\tau}{s_{4}}\right)^{\frac{3}{2}} \sum_{\nu \mid N} \frac{1}{N^{\prime}} \nu^{-\frac{1}{2}} d\left(s_{4}\right) \\
& =\left(\frac{\tau}{s_{4}}\right)^{\frac{3}{2}} \frac{1}{N} \sigma_{1 / 2}(N) d\left(s_{4}\right) \ll N^{-\frac{1}{2}+\varepsilon}\left(\frac{\tau}{s_{4}}\right)^{\frac{3}{2}} s_{4}^{\varepsilon},
\end{aligned}
$$

where $\sigma_{1 / 2}(N)=\sum_{d \mid N} d^{1 / 2}$. The proof of (4.10) is similar, but the condition $k \geq 3$ is necessary in the course of the proof to assure the convergence of a relevant series.

Using (4.9) and $A\left(s_{4}, T\right) \ll s_{4}^{\varepsilon}$, we have

$$
\begin{aligned}
\sum_{c_{1}=1}^{\infty} S_{1} & \ll|T|^{-\frac{1}{4}} \sum_{s_{4} \leq \tau / N} \sum_{c_{1} \leq \tau / s_{4} N}\left(s_{4}, N c_{1}\right)^{\frac{1}{2}}\left(N c_{1}\right)^{\frac{1}{2}} s_{4}^{\varepsilon} \\
& \ll|T|^{-\frac{1}{4}} N^{-\frac{1}{2}+\varepsilon} \sum_{s_{4} \leq \tau / N}\left(\frac{\tau}{s_{4}}\right)^{\frac{3}{2}} s_{4}^{\varepsilon} \\
& \ll|T|^{\frac{1}{2}} N^{-\frac{1}{2}+\varepsilon} .
\end{aligned}
$$


Also, using (4.10),

$$
\begin{aligned}
\sum_{c_{1}=1}^{\infty} S_{2} & \ll|T|^{\frac{k}{2}-\frac{3}{4}} \sum_{s_{4}=1}^{\infty} s_{4}^{-k+1+\varepsilon} \sum_{c_{1}>\tau / s_{4} N}\left(s_{4}, N c_{1}\right)^{\frac{1}{2}}\left(N c_{1}\right)^{\frac{3}{2}-k} \\
& \ll|T|^{\frac{k}{2}-\frac{3}{4}} N^{-\frac{1}{2}+\varepsilon} \sum_{s_{4}=1}^{\infty} s_{4}^{-k+1+\varepsilon}\left(\frac{\tau}{s_{4}}\right)^{\frac{5}{2}-k} \\
& \ll|T|^{\frac{1}{2}} N^{-\frac{1}{2}+\varepsilon} .
\end{aligned}
$$

Therefore, from (4.8) we obtain

Proposition 4.5. If $k \geq 3$, then

$$
\Sigma_{1} \ll|T|^{\frac{k}{2}-\frac{1}{4}} N^{-\frac{1}{2}+\varepsilon}
$$

\section{THE CASE OF $\operatorname{rank} C=2$}

The basic fact for the case $\operatorname{rank} C=2$ is the following lemma, which is Kitaoka's Lemma 5 ([5], p.159) when $N=1$.

Lemma 5.1. As $\mathfrak{H}_{N}^{(2)}$, we can choose

$$
\left\{M=\left(\begin{array}{cc}
* & * \\
N C & D
\end{array}\right) \in \operatorname{Sp}(2, \mathbb{Z})|| C \mid \neq 0, D \bmod N C \Lambda\right\}
$$

and $\theta(M)=\{0\}$.

The condition $\operatorname{rank} C=2$ is equivalent to $|C| \neq 0$. For the set of such matrices, Kitaoka proved:

Lemma 5.2 (Kitaoka [5], p.164, Lemma 1).

$$
\begin{aligned}
& \left\{C \in M_{2}(\mathbb{Z})|| C \mid \neq 0\right\} \\
& =\left\{U^{-1} \operatorname{diag}\left(c_{1}, c_{2}\right) V^{-1}\left|U \in \mathrm{GL}(2, \mathbb{Z}), V \in \mathrm{GL}(2, \mathbb{Z}) / P\left(c_{2} / c_{1}\right), 0<c_{1}\right| c_{2}\right\}
\end{aligned}
$$

where $\operatorname{diag}\left(c_{1}, c_{2}\right)=\left(\begin{array}{cc}c_{1} & 0 \\ 0 & c_{2}\end{array}\right)$ and

$$
P(n)=\left\{\left(\begin{array}{ll}
a & b \\
c & d
\end{array}\right) \in \mathrm{GL}(2, \mathbb{Z}) \mid b \equiv 0 \bmod n\right\} .
$$

The starting point of the argument is another formula of Kitaoka, stated in p.166 of [5], which is

$$
\begin{aligned}
h_{Q}(M, T)= & 2^{-1} \pi^{-4}\left(\frac{|T|}{|Q|}\right)^{\frac{k}{2}-\frac{3}{4}}\|N C\|^{-\frac{3}{2}} e\left(\operatorname{tr}\left(A C^{-1} Q+C^{-1} D T\right) / N\right) \\
& \times \int_{0}^{1} \prod_{i=1}^{2} J_{k-\frac{3}{2}}\left(4 \pi s_{i} u\right) u\left(1-u^{2}\right)^{-\frac{1}{2}} d u,
\end{aligned}
$$

where $s_{1}$ and $s_{2}$ are positive numbers such that $s_{1}^{2}$ and $s_{2}^{2}$ are the eigenvalues of the matrix $P_{0}=T\left[\sqrt{Q\left[{ }^{t}(N C)^{-1}\right]}\right]$. (The symbol $\|N C\|$ means simply the absolute value of the 
determinant $|N C|$.) Then

$$
\begin{aligned}
\sum_{D \bmod N C \Lambda} h_{Q}(M, T)= & 2^{-1} \pi^{-4}\left(\frac{|T|}{|Q|}\right)^{\frac{k}{2}-\frac{3}{4}}\|N C\|^{-\frac{3}{2}} K(Q, T ; N C) \\
& \times \int_{0}^{1} \prod_{i=1}^{2} J_{k-\frac{3}{2}}\left(4 \pi s_{i} u\right) u\left(1-u^{2}\right)^{-\frac{1}{2}} d u
\end{aligned}
$$

where

$$
K(Q, T ; C)=\sum_{D} e\left(\operatorname{tr}\left(A C^{-1} Q+C^{-1} D T\right)\right)
$$

with $D$ running over

$$
\left\{D(\bmod C \Lambda) \in M_{2}(\mathbb{Z}) \mid\left(\begin{array}{ll}
A & B \\
C & D
\end{array}\right) \in \mathrm{Sp}(2, \mathbb{Z})\right\} .
$$

This $K(Q, T ; C)$ is a kind of generalized Kloosterman's sum, introduced and studied by Kitaoka [5]. In particular, Kitaoka proved:

Lemma 5.3 (Kitaoka [5], p.150, Proposition 1). Let $C \in M_{2}(\mathbb{Z})$ such that $|C| \neq 0$ and $C=U^{-1}\left(\begin{array}{cc}c_{1} & 0 \\ 0 & c_{2}\end{array}\right) V^{-1}, U, V \in \mathrm{GL}(2, \mathbb{Z}), 0<c_{1} \mid c_{2}$. Then for $P, T \in \Lambda^{*}$ we have

$$
K(P, T ; C)=O\left(c_{1}^{2} c_{2}^{\frac{1}{2}+\varepsilon}\left(c_{2}, t_{4}\right)^{\frac{1}{2}}\right),
$$

where $\varepsilon$ is any positive number and $t_{4}$ is the $(2,2)$-entry of $T[V]$. Moreover, $K(P, T ; C)=$ $K\left(T, P ;{ }^{t} C\right)$ holds.

By this lemma, we find that the right-hand side of (5.1) is

$$
\left.\ll|T|^{\frac{k}{2}-\frac{3}{4}}|| N C\right|^{-\frac{3}{2}}\left(N c_{1}\right)^{2}\left(N c_{2}\right)^{\frac{1}{2}+\varepsilon}\left(N c_{2}, t_{4}\right)^{\frac{1}{2}}\left|\int_{0}^{1} \prod_{i=1}^{2} J_{k-\frac{3}{2}}\left(4 \pi s_{i} u\right) u\left(1-u^{2}\right)^{-\frac{1}{2}} d u\right|
$$

(because $Q$ is fixed).

Kitaoka ([5], p.166) showed that

$$
\int_{0}^{1} \prod_{i=1}^{2} J_{k-\frac{3}{2}}\left(4 \pi s_{i} u\right) u\left(1-u^{2}\right)^{-\frac{1}{2}} d u \ll \begin{cases}(\mathrm{a}) & |P|^{\frac{k}{2}-\frac{3}{4}} \\ \text { (b) } & |P|^{-\frac{1}{4}}, \\ (\mathrm{c}) & |P|^{\frac{k}{2}-\frac{3}{4}}(\operatorname{tr}(P))^{\frac{1-k}{2}}\end{cases}
$$

where $\left.P=T \cdot Q^{[t}(N C)^{-1}\right]$. Kitaoka stated the above (a) in case $\operatorname{tr}(P)<1$, (b) in case $\operatorname{tr}(P)<2|P|$, and (c) otherwise (which are sufficient for his purpose), but actually the above estimates themselves are valid without such conditions. This is because the estimates (4.7) are true for any $x>0$. In fact, applying (4.7) (i) to the both Bessel factors of the left-hand side of (5.3), and noting $\left(s_{1} s_{2}\right)^{2}=\left|P_{0}\right|=|P|$, we obtain the estimate (a). Applying (4.7) (ii) to the both Bessel factors we obtain (b). Applying (4.7) (i) to the Bessel factor with smaller eigenvalue $s_{i}$, and applying (4.7) (ii) to the other Bessel factor, we obtain (c).

We first use only (a) and (c) of (5.3) to obtain an estimate which is sharp with respect to $N$. It is possible to find a suitable $U_{1} \in \mathrm{GL}(2, \mathbb{Z})$ for which $A=T\left[V \operatorname{diag}\left(c_{1}, c_{2}\right)^{-1} U_{1}\right]$ is Mikowski-reduced. We may write $C$ in Lemma 5.2 as

$$
C=U^{-1} U_{1}^{-1} \operatorname{diag}\left(c_{1}, c_{2}\right) V^{-1} \text {. }
$$

Then we have

$$
|P|=\left|T \cdot Q\left[{ }^{t}(N C)^{-1}\right]\right|=N^{-4}|Q| \cdot|A| \asymp N^{-4}|A|
$$


and

$$
\left.\operatorname{tr} P=\operatorname{tr}\left(T \cdot Q\left[{ }^{t}(N C)^{-1}\right]\right) \asymp \operatorname{tr}\left(T \cdot 1_{2}{ }^{t}(N C)^{-1}\right]\right)=N^{-2} \operatorname{tr}(A[U]) .
$$

Therefore from (5.2) we have

$$
\begin{aligned}
& \sum_{U \in \mathrm{GL}(2, \mathbb{Z})}\left|\sum_{D \bmod N C \Lambda} h_{Q}(M, T)\right| \\
& \left.\ll|T|^{\frac{k}{2}-\frac{3}{4}}|| N C\right|^{-\frac{3}{2}}\left(N c_{1}\right)^{2}\left(N c_{2}\right)^{\frac{1}{2}+\varepsilon}\left(N c_{2}, t_{4}\right)^{\frac{1}{2}}\left\{\sum_{U \in \mathrm{GL}(2, \mathbb{Z}), \operatorname{tr}(A[U]) \ll 1}\left(N^{-4}|A|\right)^{\frac{k}{2}-\frac{3}{4}}\right. \\
& \left.+\sum_{U \in \operatorname{GL}(2, \mathbb{Z}), \operatorname{tr}(A[U]) \ll|A|}\left(N^{-4}|A|\right)^{\frac{k}{2}-\frac{3}{4}}+\sum_{U \in \mathrm{GL}(2, \mathbb{Z}), \text { otherwise }}\left(N^{-4}|A|\right)^{\frac{k}{2}-\frac{3}{4}}\left(N^{-2} \operatorname{tr}(A[U])\right)^{\frac{1-k}{2}}\right\},
\end{aligned}
$$

where we applied (5.3) (a) to the first and third sums, and (c) to the second sum.

Kitaoka proved ([5], p.167, Lemma 2) that if $A$ is Minkowski-reduced, then

$$
\begin{aligned}
& \sum_{U \in \mathrm{GL}(2, \mathbb{Z}), \operatorname{tr}(A[U]) \ll 1}|A|^{\frac{k}{2}-\frac{3}{4}}+\sum_{U \in \mathrm{GL}(2, \mathbb{Z}), \operatorname{tr}(A[U]) \ll|A|}|A|^{-\frac{1}{4}}+\sum_{U \in \mathrm{GL}(2, \mathbb{Z}), \text { otherwise }}|A|^{\frac{k}{2}-\frac{3}{4}} \operatorname{tr}(A[U])^{\frac{1-k}{2}} \\
& \ll m(A)^{\varepsilon} \max (1,|A|)^{\frac{3-k}{2}+\varepsilon}|A|^{\frac{k}{2}-\frac{5}{4}-\varepsilon},
\end{aligned}
$$

where $m(A)=\min \left\{A[x] \mid x \in \mathbb{Z}^{2}, x \neq(0,0)\right\}$. Using (5.5), we find that the first and the third sums on the right-hand side of (5.4) are

$$
\begin{aligned}
& \ll\left(N^{-2 k+3}+N^{-k+2}\right) m(A)^{\varepsilon} \max (1,|A|)^{\frac{3-k}{2}+\varepsilon}|A|^{\frac{k}{2}-\frac{5}{4}-\varepsilon} \\
& \ll N^{-k+2} m(A)^{\varepsilon} \max (1,|A|)^{\frac{3-k}{2}+\varepsilon}|A|^{\frac{k}{2}-\frac{5}{4}-\varepsilon} .
\end{aligned}
$$

On the other hand, in the proof of the above Lemma 2 of Kitaoka, he proved ([5], p.168, line 7) the following

\section{Lemma 5.4.}

$$
\begin{aligned}
\#\{U \in \mathrm{GL}(2, \mathbb{Z})|\operatorname{tr}(A[U]) \ll| A \mid\} & \ll \#\{U \in \mathrm{GL}(2, \mathbb{Z})|\operatorname{tr}(H[U]) \ll| H \mid\} \\
& \ll c^{\frac{1}{2}} a^{\frac{1}{2}+\varepsilon}
\end{aligned}
$$

where we set $H=\left(\begin{array}{ll}a & 0 \\ 0 & c\end{array}\right)$ for $A=\left(\begin{array}{ll}a & b \\ b & c\end{array}\right)$. In particular,

$$
\#\{U \in \mathrm{GL}(2, \mathbb{Z})|\operatorname{tr}(A[U]) \ll| A \mid\} \ll|A|^{\frac{1}{2}+\varepsilon}
$$

if $A$ is Minkowski-reduced.

Therefore, the second sum on the right-hand side of (5.4) is

$$
\ll N^{-2 k+3}|A|^{\frac{k}{2}-\frac{3}{4}} \sum_{U \in \mathrm{GL}(2, \mathbb{Z}), \operatorname{tr}(A[U]) \ll|A|} 1 \ll N^{-2 k+3}|A|^{\frac{k}{2}-\frac{1}{4}+\varepsilon} .
$$


Collecting the above estimates, and noting $\|N C\|=N^{2}\left|c_{1} c_{2}\right|$, from (5.4) we obtain

$$
\begin{aligned}
& \sum_{U \in \mathrm{GL}(2, \mathbb{Z})}\left|\sum_{D \bmod N C \Lambda} h_{Q}(M, T)\right| \ll|T|^{\frac{k}{2}-\frac{3}{4}} N^{-\frac{1}{2}+\varepsilon}\left|c_{1}\right|^{\frac{1}{2}}\left|c_{2}\right|^{-1+\varepsilon}\left(N c_{2}, t_{4}\right)^{\frac{1}{2}} \\
& \times\left\{N^{-2 k+3}|A|^{\frac{k}{2}-\frac{1}{4}+\varepsilon}+N^{-k+2} m(A)^{\varepsilon} \max (1,|A|)^{\frac{3-k}{2}+\varepsilon}|A|^{\frac{k}{2}-\frac{5}{4}-\varepsilon}\right\} .
\end{aligned}
$$

Similarly to (4.4), we see that $t_{4}=T[v]$, where $v$ is the vector consisting of the second column of $V$. Using this fact, $|A|=|T|\left(c_{1} c_{2}\right)^{-2}$ and $\left(N c_{2}, T[v]\right)^{\frac{1}{2}} \leq N^{\frac{1}{2}}\left(c_{2}, T[v]\right)^{\frac{1}{2}}$, we find that the right-hand side of (5.6) is

$$
\begin{aligned}
\ll & |T|^{\frac{k}{2}-\frac{3}{4}} N^{\varepsilon} c_{1}^{\frac{1}{2}} c_{2}^{-1+\varepsilon}\left(c_{2}, T[v]\right)^{\frac{1}{2}}\left\{N^{-2 k+3}|T|^{\frac{k}{2}-\frac{1}{4}+\varepsilon}\left(c_{1} c_{2}\right)^{-k+\frac{1}{2}+\varepsilon}\right. \\
& \left.+N^{-k+2} m\left(T\left[V \operatorname{diag}\left(c_{1}, c_{2}\right)^{-1} U_{1}^{-1}\right]\right)^{\varepsilon} \cdot \max \left(1,|T|\left(c_{1} c_{2}\right)^{-2}\right)^{\frac{3-k}{2}+\varepsilon} \cdot|T|^{\frac{k}{2}-\frac{5}{4}-\varepsilon}\left(c_{1} c_{2}\right)^{-k+\frac{5}{2}+\varepsilon}\right\} .
\end{aligned}
$$

Therefore,

$$
\begin{aligned}
\sum_{0<c_{1} \mid c_{2}} \sum_{V \in \mathrm{GL}(2, \mathbb{Z}) / P\left(c_{1} / c_{2}\right)} \sum_{U \in \mathrm{GL}(2, \mathbb{Z})}\left|\sum_{D \bmod N C \Lambda} h_{Q}(M, T)\right| \\
=\sum_{0<c_{1} \mid c_{2}} \sum_{V \in \mathrm{GL}(2, \mathbb{Z}) / P\left(c_{1} / c_{2}\right)}\left\{W_{1}\left(c_{1}, c_{2}, V\right)+W_{2}\left(c_{1}, c_{2}, V\right)\right\},
\end{aligned}
$$

where

$$
\begin{aligned}
W_{1}\left(c_{1}, c_{2}, V\right)= & |T|^{\frac{k}{2}-\frac{3}{4}} N^{-2 k+3+\varepsilon} c_{1}^{-k+1+\varepsilon} c_{2}^{-k-\frac{1}{2}+\varepsilon}\left(c_{2}, T[v]\right)^{\frac{1}{2}}|T|^{\frac{k}{2}-\frac{1}{4}+\varepsilon} \\
W_{2}\left(c_{1}, c_{2}, V\right)= & |T|^{\frac{k}{2}-\frac{3}{4}} N^{-k+2+\varepsilon} c_{1}^{-k+3+\varepsilon} c_{2}^{-k+\frac{3}{2}+\varepsilon}\left(c_{2}, T[v]\right)^{\frac{1}{2}} m\left(T\left[V \operatorname{diag}\left(c_{1}, c_{2}\right)^{-1} U_{1}^{-1}\right]\right)^{\varepsilon} \\
& \times \max \left(1,|T|\left(c_{1} c_{2}\right)^{-2}\right)^{\frac{3-k}{2}+\varepsilon} \cdot|T|^{\frac{k}{2}-\frac{5}{4}-\varepsilon} .
\end{aligned}
$$

The form of $W_{2}\left(c_{1}, c_{2}, V\right)$ is quite similar to the right-hand side of Kitaoka's Lemma 3 ([5], p. 169). Therefore, by the same argument as in p.170 of Kitaoka [5], we obtain

$$
\sum_{0<c_{1} \mid c_{2}} \sum_{V \in \mathrm{GL}(2, \mathbb{Z}) / P\left(c_{1} / c_{2}\right)} W_{2}\left(c_{1}, c_{2}, V\right) \ll N^{2-k+\varepsilon}|T|^{\frac{k}{2}-\frac{1}{4}+\varepsilon} .
$$

On the other hand,

$$
\begin{aligned}
& \sum_{0<c_{1} \mid c_{2}} \sum_{V \in \mathrm{GL}(2, \mathbb{Z}) / P\left(c_{1} / c_{2}\right)} W_{1}\left(c_{1}, c_{2}, V\right) \\
& =|T|^{k-1+\varepsilon} N^{3-2 k+\varepsilon} \sum_{0<c_{1} \mid c_{2}} c_{1}^{-k+1+\varepsilon} c_{2}^{-k-\frac{1}{2}+\varepsilon} \sum_{V \in \mathrm{GL}(2, \mathbb{Z}) / P\left(c_{1} / c_{2}\right)}\left(c_{2}, T[v]\right)^{\frac{1}{2}} .
\end{aligned}
$$

To evaluate this double sum, here we quote one more result of Kitaoka. For $G=\left(g_{i j}\right) \in \Lambda^{*}$, set $e(G):=\operatorname{gcd}\left(g_{11}, g_{22}, 2 g_{12}\right)$. Let $S=\left\{\left(\begin{array}{l}b \\ d\end{array}\right) ; b, d \in \mathbb{Z},(b, d)=1\right\}$. For a positive integer $n$, define $S(n)=S / \sim$, where we denote $\left(\begin{array}{l}b \\ d\end{array}\right) \sim\left(\begin{array}{l}b^{\prime} \\ d^{\prime}\end{array}\right)$ if there exists a $w \in \mathbb{Z}$ such that $(w, n)=1$ and $\left(\begin{array}{l}b \\ d\end{array}\right) \equiv w\left(\begin{array}{l}b^{\prime} \\ d^{\prime}\end{array}\right)(\bmod n)$. 
Lemma 5.5 (Kitaoka [5], p.154, Proposition 2). For any $P \in \Lambda^{*}$, we have

$$
\sum_{x \in S(n)}(P[x], n)^{\frac{1}{2}}=O\left(n^{1+\varepsilon}(e(P), n)^{\frac{1}{2}}\right) .
$$

Applying this lemma to $P[x]=T[v], n=c_{2} / c_{1}$. Then

$$
\begin{aligned}
\sum_{V \in \operatorname{GL}(2, \mathbb{Z}) / P\left(c_{2} / c_{1}\right)}\left(c_{2}, T[v]\right)^{\frac{1}{2}} & \leq \sum_{V \in \operatorname{GL}(2, \mathbb{Z}) / P\left(c_{2} / c_{1}\right)} c_{1}^{\frac{1}{2}}\left(c_{2} / c_{1}, T[v]\right)^{\frac{1}{2}} \\
& \ll c_{1}^{\frac{1}{2}}\left(c_{2} / c_{1}\right)^{1+\varepsilon}\left(c_{2} / c_{1}, T[v]\right)^{\frac{1}{2}} \\
& \ll c_{1}^{\frac{1}{2}}\left(c_{2} / c_{1}\right)^{\frac{3}{2}+\varepsilon},
\end{aligned}
$$

where the second inequality follows from the lemma and the fact $e(T) \leq t_{4}=T[v]$. Therefore we have

$$
\begin{aligned}
& \sum_{0<c_{1} \mid c_{2}} c_{1}^{-k+1+\varepsilon} c_{2}^{-k-\frac{1}{2}+\varepsilon} \sum_{V \in \operatorname{GL}(2, \mathbb{Z}) / P\left(c_{2} / c_{1}\right)}\left(c_{2}, T[v]\right)^{\frac{1}{2}} \\
& \ll \sum_{0<c_{1} \mid c_{2}} c_{1}^{-k+\varepsilon} c_{2}^{-k+1+\varepsilon}=\sum_{c_{1}=1}^{\infty} \sum_{c_{3}=1}^{\infty} c_{1}^{-2 k+1+\varepsilon} c_{3}^{-k+1+\varepsilon},
\end{aligned}
$$

where we put $c_{2}=c_{1} c_{3}$. This is convergent when $k \geq 3$. Then from (5.9) we have

$$
\sum_{0<c_{2} \mid c_{1}} \sum_{V \in \mathrm{GL}_{2}(\mathbb{Z}) / P\left(c_{2} / c_{1}\right)} W_{1}\left(c_{1}, c_{2}, V\right) \ll|T|^{k-1+\varepsilon} N^{3-2 k+\varepsilon} .
$$

Substituting (5.8) and (5.10) into the right-hand side of (5.7), we now obtain

Proposition 5.6. If $k \geq 3$, then

$$
\Sigma_{2} \ll N^{2-k+\varepsilon}|T|^{\frac{k}{2}-\frac{1}{4}+\varepsilon}+N^{3-2 k+\varepsilon}|T|^{k-1+\varepsilon} .
$$

Next, we make use of (5.3) (b) as well as (a) and (c) to deduce another estimate of $\Sigma_{2}$. This time, instead of $A$, we use $A^{\prime}=T\left[V \operatorname{diag}\left(N c_{1}, N c_{2}\right)^{-1} U_{1}\right]$. Then $|P| \asymp\left|A^{\prime}\right|, \operatorname{tr} P \asymp \operatorname{tr}\left(A^{\prime}[U]\right)$, and hence the sums in the curly parentheses on the right-hand side of (5.4) are replaced by

$$
\begin{aligned}
& \sum_{U \in \mathrm{GL}(2, \mathbb{Z}), \operatorname{tr}\left(A^{\prime}[U]\right) \ll 1}\left|A^{\prime}\right|^{\frac{k}{2}-\frac{3}{4}}+\sum_{U \in \mathrm{GL}(2, \mathbb{Z}), \operatorname{tr}\left(A^{\prime}[U]\right) \ll\left|A^{\prime}\right|}\left|A^{\prime}\right|^{-\frac{1}{4}} \\
& +\sum_{U \in \mathrm{GL}(2, \mathbb{Z}), \text { otherwise }}\left|A^{\prime}\right|^{\frac{k}{2}-\frac{3}{4}}\left(\operatorname{tr}\left(A^{\prime}[U]\right)\right)^{\frac{1-k}{2}},
\end{aligned}
$$

for which (5.5) can be directly applied. Therefore

$$
\begin{aligned}
& \sum_{0<c_{1} \mid c_{2}} \sum_{V \in \mathrm{GL}(2, \mathbb{Z}) / P\left(c_{1} / c_{2}\right)} \sum_{U \in \mathrm{GL}(2, \mathbb{Z})}\left|\sum_{D \bmod N C \Lambda} h_{Q}(M, T)\right| \\
& \left.\ll \sum_{0<c_{1} \mid c_{2}} \sum_{V \in \mathrm{GL}(2, \mathbb{Z}) / P\left(c_{1} / c_{2}\right)}|T|^{\frac{k}{2}-\frac{3}{4}}|| N C\right|^{-\frac{3}{2}}\left(N c_{1}\right)^{2}\left(N c_{2}\right)^{\frac{1}{2}+\varepsilon}\left(N c_{2}, t_{4}\right)^{\frac{1}{2}} \\
& \times m\left(A^{\prime}\right)^{\varepsilon} \max \left(1,\left|A^{\prime}\right|\right)^{\frac{3-k}{2}+\varepsilon}\left|A^{\prime}\right|^{\frac{k}{2}-\frac{5}{4}-\varepsilon} \\
& \ll N^{-2 k+\frac{9}{2}+\varepsilon}|T|^{k-2+\varepsilon} \sum_{0<c_{1} \mid c_{2} V \in \mathrm{GL}(2, \mathbb{Z}) / P\left(c_{1} / c_{2}\right)} c_{1}^{-k+3+\varepsilon} c_{2}^{-k+\frac{3}{2}+\varepsilon} \\
& \times\left(N c_{2}, T[v]\right)^{\frac{1}{2}} \max \left(1, N^{-4}|T|\left(c_{1} c_{2}\right)^{-2}\right)^{\frac{3-k}{2}+\varepsilon} \\
& =R_{1}+R_{2},
\end{aligned}
$$


say, where $R_{1}$ denotes the part with $\left(c_{1} c_{2}\right)^{2} \geq N^{-4}|T|$, and $R_{2}$ the remaining part. Then

$$
\begin{aligned}
R_{2} & =N^{-\frac{3}{2}+\varepsilon}|T|^{\frac{k}{2}-\frac{1}{2}+\varepsilon} \sum_{\substack{0<c_{1}\left|c_{2} \\
\left(c_{1} c_{2}\right)^{2}<N^{-4}\right| T \mid}} c_{2}^{-\frac{3}{2}+\varepsilon} \sum_{V \in \mathrm{GL}(2, \mathbb{Z}) / P\left(c_{1} / c_{2}\right)}\left(N c_{2}, T[v]\right)^{\frac{1}{2}} \\
& \ll N^{-\frac{3}{2}+\varepsilon}|T|^{\frac{k}{2}-\frac{1}{2}+\varepsilon} \sum_{\substack{0<c_{1}\left|c_{2} \\
\left(c_{1} c_{2}\right)^{2}<N^{-4}\right| T \mid}} c_{2}^{-\frac{3}{2}+\varepsilon}\left(N c_{1}\right)^{\frac{1}{2}} \sum_{V \in \operatorname{GL}(2, \mathbb{Z}) / P\left(c_{1} / c_{2}\right)}\left(c_{2} / c_{1}, T[v]\right)^{\frac{1}{2}} \\
& \ll N^{-1+\varepsilon}|T|^{\frac{k}{2}-\frac{1}{2}+\varepsilon} \sum_{\substack{0<c_{1}\left|c_{2} \\
\left(c_{1} c_{2}\right)^{2}<N^{-4}\right| T \mid}} c_{1}^{\frac{1}{2}} c_{2}^{-\frac{3}{2}+\varepsilon}\left(\frac{c_{2}}{c_{1}}\right)^{1+\varepsilon}\left(c_{2} / c_{1}, e(T)\right)^{\frac{1}{2}}
\end{aligned}
$$

by Lemma 5.5, Putting $c_{2}=n c_{1}$, we obtain

$$
R_{2} \ll N^{-1+\varepsilon}|T|^{\frac{k}{2}-\frac{1}{2}+\varepsilon} \sum_{c_{1}=1}^{\infty} c_{1}^{-1+\varepsilon} \sum_{n<|T|^{1 / 2}\left(c_{1} N\right)^{-2}} n^{-\frac{1}{2}+\varepsilon}(n, e(T))^{\frac{1}{2}} .
$$

The last sum is $O\left(e(T)^{\varepsilon}\left(|T|^{\frac{1}{2}}\left(c_{1} N\right)^{-2}\right)^{\frac{1}{2}+\varepsilon}\right)$ as is shown in p.170 of Kitaoka [5]. Hence

$$
R_{2} \ll N^{-2+\varepsilon}|T|^{\frac{k}{2}-\frac{1}{4}+\varepsilon} e(T)^{\varepsilon} \sum_{c_{1}=1}^{\infty} c_{1}^{-2+\varepsilon} \ll N^{-2+\varepsilon}|T|^{\frac{k}{2}-\frac{1}{4}+\varepsilon} .
$$

The remaining part $R_{1}$ can be treated similarly, and it is estimated by exactly the same right-hand side as that of (5.11). Therefore we now obtain

\section{Proposition 5.7.}

$$
\Sigma_{2} \ll N^{-2+\varepsilon}|T|^{\frac{k}{2}-\frac{1}{4}+\varepsilon} .
$$

\section{Completion of the proof of Theorem 1.1}

From (2.11), Proposition 3.2, Proposition 4.3 and Proposition 5.6, we have

$$
\begin{aligned}
A_{Q, N}(T) & =\#\left\{U \in \mathrm{GL}_{2}(\mathbb{Z}) \mid U Q^{t} U=T\right\} \\
& +O\left(N^{\frac{3}{2}-k}|T|^{k-\frac{3}{2}}+N^{2-k+\varepsilon}|T|^{\frac{k}{2}-\frac{1}{4}+\varepsilon}+N^{3-2 k+\varepsilon}|T|^{k-1+\varepsilon}\right)
\end{aligned}
$$

for $k \geq 3$. This implies assertions (i) and (ii) of Theorem 1.1. On the other hand, from (2.11), Proposition [3.2, Proposition 4.5 and proposition 5.7, assertion (iii) follows. The proof of Theorem 1.1 is thus complete.

\section{REFERENCES}

[1] W. Duke, The critical order of vanishing of automorphic L-functions with large level, Invent. Math. 119 (1995), 165-174.

[2] LooKeng Hua, Introduction to Number Theory, Springer-Verlag, 1982.

[3] H. Iwaniec, Topics in Classical Automorphic Forms, Graduate Studies in Mathematics 17, Amer. Math. Soc., 1997.

[4] Y. Kamiya, Certain mean values and non-vanishing of automorphic L-functions with large level, Acta Arith. 93 (2000), 157-176.

[5] Y. Kitaoka, Fourier coefficients of Siegel cusp forms of degree two, Nagoya Math. J. 93 (1984), 149-171.

[6] H. Klingen, Introductory Lectures on Siegel Modular Forms, Cambridge studies in advanced mathematics 20, Cambridge University Press, 1990.

[7] E. Kowalski, A. Saha and J. Tsimerman, Local spectral equidistribution for Siegel modular forms and applications, to appear in Compositio Math. 
Department of Mathematics, Graduate School of Science, Kyoto University, Kyoto 6068502, JAPAN

E-mail address: chida@math.kyoto-u.ac.jp

Muroran Institute of Technology, 27-1 Mizumoto, Muroran, 050-8585, Japan

E-mail address: hidenori@mmm.muroran-it.ac.jp

Graduate School of Mathematics, Nagoya University, Chikusa-Ku, Nagoya 464-8602, Japan

E-mail address: kohjimat@math.nagoya-u.ac.jp 